\title{
Rotating Machinery Vibration Signal Processing And Fault Diagnosis Based on LMD
}

\author{
Ruirui Bo and Ze Zhang \\ Department of Electronic and Information Engineering, Inner Mongolia University, Hohhot, 10126, China
}

\begin{abstract}
There are abundant of fault information in rotating machinery vibration signal. On account of the nonlinearity and non-stationarity, the paper first does pre-process to the vibration signal using wavelet threshold denoising method and this method can bring a smooth signal. Then it decomposes the vibration signal using local mean decomposition(LMD), which is effective to the vibration signal. The LMD decomposes the signal into many PFs as the frequency from high to low. These PFs are composed of the production of envelop signal and pure frequency modulated signal. Finally, it takes most use of the kurtosis which is sensitive to the fault impact. By calculating the kurtosis of PF, it can assess the distribution of fault impact signal in every frequency band, consequently distinguishing the operating state of bearing and recognizing the fault mode according to the growth of turtosis. The experiment of actual bearing vibration signal demonstrates that the methods this paper proposed can effectively diagnose the vibration fault and has good performance.
\end{abstract}

Keywords: LMD, wavelet threshold method, kurtosis, fault diagnosis.

\section{Introduction}

Giant rotating machinery like air compressor and pump and turbine are key production equipment in modern industry. With the substantial improvement of science and technology and automation, giant rotating machinery becomes high precision and high speed and serialization and automation[1]. The interrelation and coupling between different portions in device grows much closer. Once there occurs a fault in a certain portion, it not only has influence on the operation of machinery itself but also affects the subsequent production, causing chain reaction and breakdown of system, leading to great financial loss even disastrous consequence. The fault information is usually shown through vibration signal when there is fault in a certain giant rotating machinery device[2]. It is the primary approach to supervise and diagnose the operating state of device using vibration signal at present. Due to operating environment's complexity and variety of giant rotating machinery, its vibration signal usually has strong non-linearity and nonstationarity. Therefore, the traditional fault diagnosis method based on classical signal procession like FFT and wavelet analysis cannot meet the demand on fault feature extraction, and they cannot make ends meet of local detail feature and general view feature in time and frequency domain,and which makes it an urgent theoretical study and practical application requirement to develop modern self-adaptive signal analysis method based on time-frequency analysis aiming at non-linear and non-stationary signal[3].

\section{Vibration signal pre-process}

\subsection{Vibration data acquisition}

Bearing, as the significant component in rotating machinery, would receive key information of rotating machinery system's operation state by analyzing and processing vibration signal. The vibration data used in this paper are from America Case Western Reserve University bearing data center. The experimented bearing is 6205-2RS JEM SKF deep groove ball bearing. There are four states that are normal operating and outer raceway fault and inner raceway fault as well as ball fault,all these single point faults are introduced to the test bearing using electro-discharge machining with fault diameters of $0.18 \mathrm{~mm}, 0.36 \mathrm{~mm}, 0.53 \mathrm{~mm}, 0.71 \mathrm{~mm}$, and $1.02 \mathrm{~mm}$, the paper chooses $0.53 \mathrm{~mm}$ fault. Vibration data is collected using accelerometer and at 12,000 samples per second. Motor speeds is 1797 . The driving end bears no load. The vibration signal wave of the four operating state are shown as fig.1. 


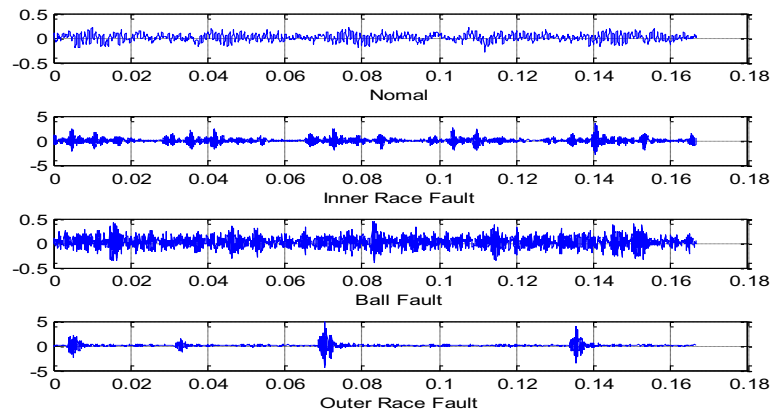

Fig.1: Vibration signal wave of the four operating state

\subsection{Wavelet threshold De-noise}

The vibration signal is corrupted by a great number of noise contribution besides fault information, which appears to be non-linear and non-stationary and with low signal noise ratio. When decomposing the signal with noise using LMD, the noise contribution may, on the one hand, affect the cyclic process and, on the other hand, bring down the timeliness but promote the decomposing time, even result in mode mixing phenomenon. It is the precondition and guarantee of signal fault diagnosis to reduce noise contribution effectively[4]. Wavelet threshold de-noise is a typical de-noising method, which has irreplaceable significance for its good time-frequency property in de-noise. The paper opts for SYM5 wavelet function and decomposes the signal to the fifth layer then uses minimaxi threshold chose to dispose coefficients for the sake of de-noise. The signal after de-noising are listed in fig. 2.

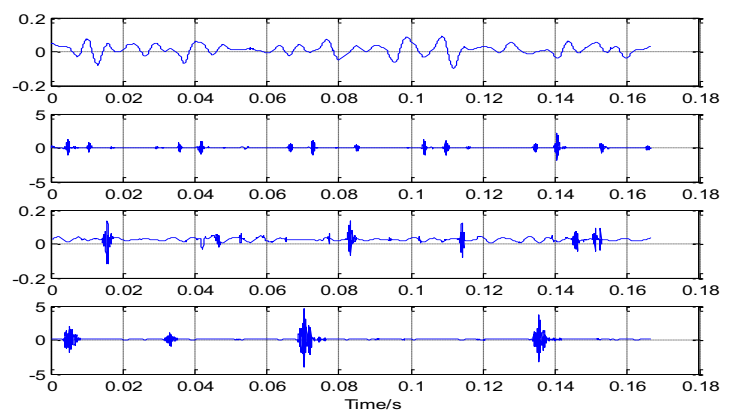

Fig.2: Denoised signal on four state

After wavelet de-noising, there is basically no noise contribution in the bearing vibration signal and the vibration is much smoother. From fig.2, it can see the real vibration signal. Analysis of the de-noised signal suggests that there are different levels of impact in the signal which is the main reflect of fault when operating.

\section{Local Mean Decomposition}

Essentially the LMD scheme involves progressively separating a frequency modulated signal from an amplitude modulated envelope signal. This separation is achieved by smoothing the original signal, subtracting the smoothed signal from the original signal, and then amplitude demodulating the result using an envelope estimate. The envelope estimate and the smoothed version of the original signal are both obtained by using moving averaging weighted by the time-lapse between the successive extrema of the original signal[5]. The instantaneous frequency can then be calculated from the frequency modulated signal. The corresponding envelope estimates are multiplied together to form a final envelope. This envelope is then multiplied by the frequency modulated signal to form a product function, which is subtracted from the original signal. The whole process is then repeated on the resulting signal, to produce a second product function, with an associated envelope and instantaneous frequency.This decomposition continues until the remaining signal contains no more oscillations.The resulting instantaneous frequency and envelope values can be plotted together in the form of a demodulated signal time-frequency representation[6]. For a certain non-stationary signal $x(\mathrm{t})$, the specific decomposing process is as follow:

Step 1: Searching for the local maximum(minimum)value point of the signal $n_{i}$, then calculating the mean value of the adjacent extremum.

$$
m_{i}=\frac{n_{i}+n_{i+1}}{2}
$$

Connecting the adjacent mean value point $m_{i}$ with straight line, smoothing the straight line using moving average method and obtaining the local mean function $m_{11}(\mathrm{t})$.

Step 2: Calculating the envelope estimate:

$$
a_{i}=\frac{\left|n_{i}-n_{i+1}\right|}{2}
$$

Connecting the adjacent envelope estimate point $a_{i}$ with straight line, smoothing the straight line using moving average method and obtaining the envelope estimate function $a_{11}(\mathrm{t})$.

Step 3: Separating the local mean function $m_{11}(\mathrm{t})$ from the original signal $x(\mathrm{t})$.

$$
h_{11}(\mathrm{t})=x(\mathrm{t})-m_{11}(\mathrm{t})
$$

Step 4: Using the envelope estimate function $a_{11}(\mathrm{t})$ to demodulate $h_{11}(\mathrm{t})$.

$$
s_{11}(\mathrm{t})=\frac{h_{11}(\mathrm{t})}{a_{11}(\mathrm{t})}
$$

The ideal situation is that $s_{11}(\mathrm{t})$ is pure frequencymodulated signal now, i.e. envelope estimate function $a_{12}(\mathrm{t})=1$. If not, taking $s_{11}(\mathrm{t})$ as original signal and repeating the step1-step4 until $s_{1 \mathrm{n}}(\mathrm{t})$ 's envelope estimate function $a_{1(\mathrm{n}+1)}(\mathrm{t})=1$, i.e. $s_{1 \mathrm{n}}(\mathrm{t})$ is a pure frequency-modulated signal. But in general it cannot meet the ideal condition, so it sets a reference quantity $\Delta$, when $1-\Delta \leq a_{1 n}(\mathrm{t}) \leq 1+\Delta$, the iteration stops. 
Step 5: Calculating the envelope signal (instantaneous amplitude function):

$$
a_{1}(\mathrm{t})=a_{11}(\mathrm{t}) a_{12}(\mathrm{t}) \ldots a_{1 n}(\mathrm{t})=\prod_{q=1}^{n} a_{1 q}(\mathrm{t})
$$

Step 6: Obtaining the first PF component by multiplying envelope signal $a_{1}(\mathrm{t})$ and pure frequencymodulated signal $s_{1 \mathrm{n}}(\mathrm{t})$.

$$
P_{1}(\mathrm{t})=a_{1}(\mathrm{t}) \mathrm{s}_{1 n}(\mathrm{t})
$$

It contains the highest frequency component of original signal, $P_{1}(\mathrm{t})$ is a simple component amplitudemodulated and frequency-modulated signal, $a_{1}(\mathrm{t})$ is the instantaneous amplitude of PF, its instantaneous frequency $f_{1}(\mathrm{t})$ can be calculated from pure frequencymodulated signal.

$$
f_{1}(\mathrm{t})=\frac{1}{2 \pi} \frac{d\left[\arccos \left(\mathrm{s}_{1 n}(\mathrm{t})\right)\right]}{d t}
$$

Step 7: After separating the first PF component from the original signal $x(\mathrm{t})$, it obtains the new signal $u_{1}(\mathrm{t})$.
Taking $u_{1}(\mathrm{t})$ as the original signal and repeat step1-step6 for $\mathrm{k}$ times until $u_{k}(\mathrm{t})$ is the monotone function.

$$
\left\{\begin{array}{l}
u_{1}(\mathrm{t})=x(\mathrm{t})-P_{1}(\mathrm{t}) \\
u_{2}(\mathrm{t})=u_{1}(\mathrm{t})-P_{2}(\mathrm{t}) \\
\vdots \\
u_{k}(\mathrm{t})=u_{k-1}(\mathrm{t})-P_{k}(\mathrm{t})
\end{array}\right.
$$

Ultimately, the original signal $x(\mathrm{t})$ can be expressed by all PF components and the residual $u_{k}(\mathrm{t})$.

$$
x(\mathrm{t})=\sum_{p=1}^{k} P_{p}(\mathrm{t})+u_{k}(\mathrm{t})
$$

Step 8: Combining the instantaneous amplitude and instantaneous frequency of all PF components again according to time-frequency-amplitude 3-D distribution.

Using the LMD to decompose the four operating state vibration signal, the PF components of each state are as fig.3-fig.6.
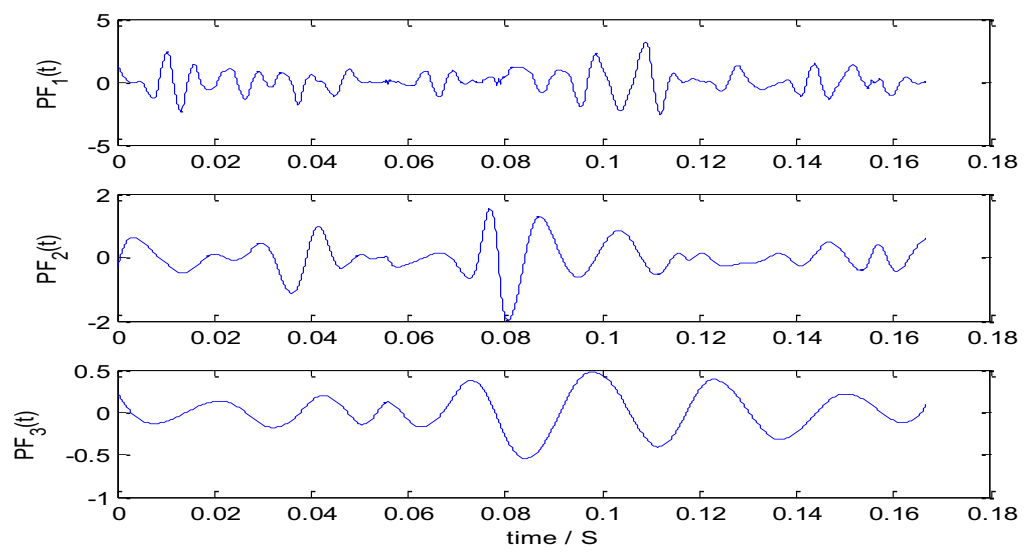

Fig.3: PF component of normal state after LMD

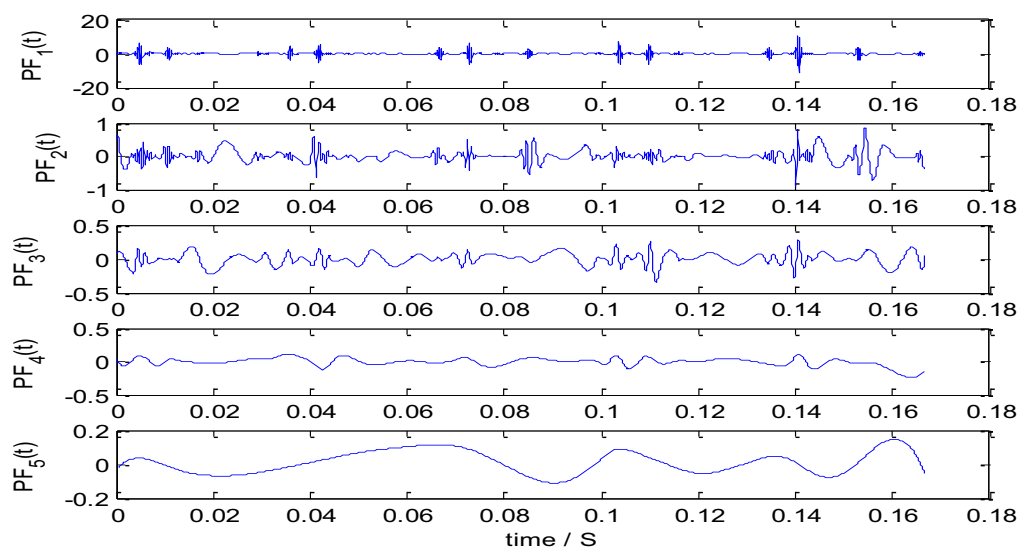

Fig.4: PF component of inner raceway fault after LMD 


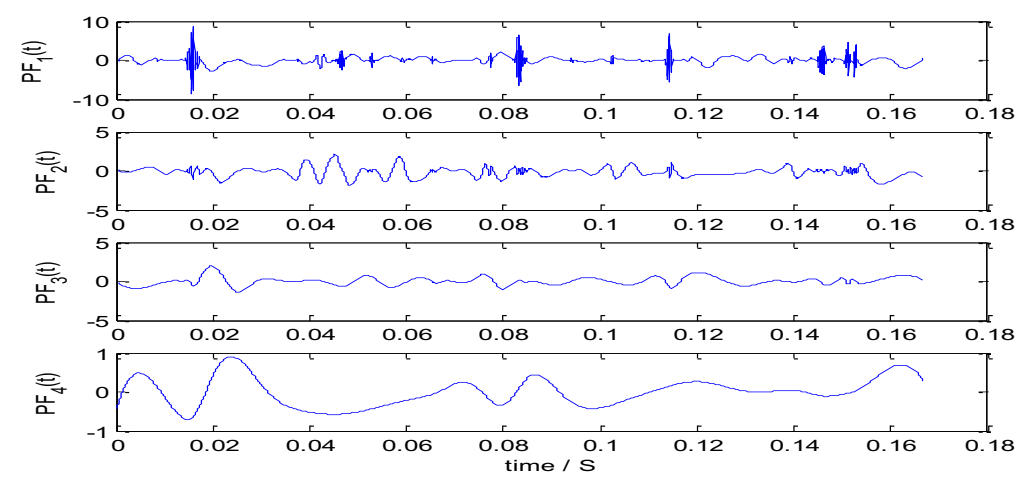

Fig.5: PF component of ball fault after LMD

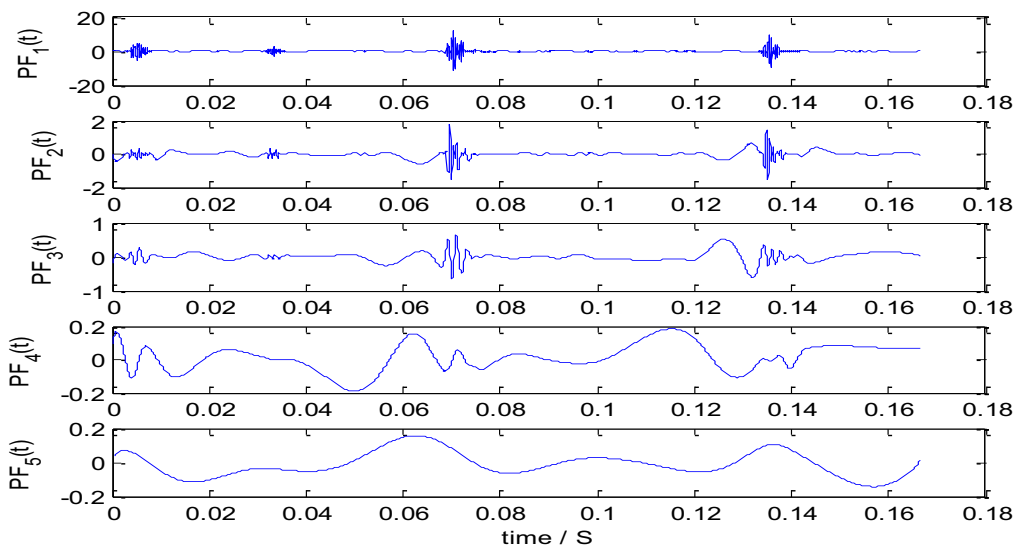

Fig.6: PF component of outer raceway fault after LMD

\section{Rotating bearing fault diagnose based on PF kurtosis}

Diagnosis information acquisition as well as fault feature extraction and state recognition are fundamental content of rotating bearing's state surveillance and fault diagnosis while fault feature extraction is the most significant and difficult problem. It directly affects the accuracy of diagnosis and reliability of prediction, in a way is the key factor to implement the rotating bearing fault diagnosis.

Kurtosis is a kind of measurement of degree of peak. A big kurtosis corresponds to a sharp wave peak and suggests that the signal has impact nature. As for the rotating bearing vibration signal, sharp peaks are usually caused by fault. For this reason, the paper combines kurtosis and LMD, thereby calculating kurtosis of signal in different frequency band after LMD. When retaining the advantage of kurtosis's sensibility to the fault impact, it studies the distribution of fault impact signal frequency in different frequency band.

Kurtusis is a statistic reflecting degree of peak, it describes the distribution character of vibration signal. The definition of kurtosis $\mathrm{K}$ is:

$$
K=\frac{E(x-\mu)^{4}}{\sigma^{4}}
$$

Here, $\mu$ is the mean of signal $\mathrm{x}, \sigma$ is the standard deviation of signal $\mathrm{x}, E(t)$ is the value of expectation of variable $t$ [7].
In general, fault information is usually included in high frequency signal, as a consequence, the paper opts for the first three PF components of the four state after LMD and calculates their kurtosis in the form of table I.

Table 1: Kurtosis of PF on four state

\begin{tabular}{|c|c|c|c|}
\hline & PF1 & PF2 & PF3 \\
\hline Normal Data & 4.092 & 5.400 & 2.727 \\
\hline $\begin{array}{c}\text { Inner Race } \\
\text { Fault }\end{array}$ & 35.300 & 6.283 & 3.371 \\
\hline Ball Data & 14.200 & 8.866 & 4.190 \\
\hline $\begin{array}{c}\text { Outer Race } \\
\text { Fault }\end{array}$ & 54.350 & 15.350 & 7.070 \\
\hline
\end{tabular}

From the table, it can be known that when the bearing operating in normal state, there is no impact caused by fault, its vibration signal is relatively steady and kurtosis is comparatively small. When fault occurs, period impact character appears in the vibration signal, the kurtosis of $\mathrm{PF}$ containing main fault information increase in large scale. Kurtosis of PF1 has the most great growth, on account that there are many impact components caused by fault in these PFs. Impact from different types of fault has different distribution in PF, thus the kurtosis are in different values just as shown in table I. The kurtosis of PF1 on inner raceway fault increases 9 times, the ball fault is 3.5 times and outer raceway fault 13 times.Therefore the paper determines the fault mode of rotating bearing from the scale of kurtosis increase. 


\section{Conclusions}

Wavelet threshold de-noise can remove the noise contribution effectively from vibration signal and expose the real signal to light. LMD has well self-adaptability when processing non-linear and non-stationary signal, and it decomposes the vibration signal into PF components with variety frequency. By calculating the kurtosis of every PF component, it can directly assess the existence of impact in PF component signal with quantification and knows the operating state of bearing from kurtosis. Moreover, it can determines the mode of fault from the growth of kurtosis. Actual vibration signal experiment proves that the fault analysis based on LMD vibration signal process has good performance.

\section{Acknowledgements}

The authors of the paper would like to thank College of Electronic and Information to provide us with equipment to finish the experiment and the paper. We also appreciate our classmates in Control Engineer Laboratory for technical support and helpful discussions.

\section{References}

1. Y. Amirat, M. Benbouzid, T. Wang, \& S. Turri, Performance analysis of an EEMD-based hilbert transform as a bearing failure detector in wind turbines. 2014 First International Conference on Green Energy ICGE, 193-198, (2014).

2. Z. Junhong \& L. Yu, Bearing fault diagnosis based on improved LMD. 2011 International Conference on Transportation, Mechanical, and Electrical Engineering (TMEE). Changchun, China, 2544-2547, (2011).

3. X. Chen, C. Yin, \& W. He, Feature extraction of gearbox vibration signals based on EEMD and sample entropy. 2013 10th International Conference on Fuzzy Systems and Knowledge Discovery (FSKD), 811-815, (2013).

4. J. Jenitta \& A. Rajeswari, Denoising of ECG signal based on improved adaptive filter with EMD and EEMD. Proceedings of 2013 IEEE Conference on Information and Communication Technologies (ICT 2013), 957-962, (2013).

5. Z. Li, Z. Wan, X. Xiong, \& X. Liao, The application of LMD and BP neural network in gear fault diagnosis. 2013 25th Chinese Control and Decision Conference (CCDC), 4609-4612, (2013).

6. J. S Smith, The local mean decomposition and its application to EEG perception data. Journal of the Royal Society Interface, 2, 5, 443-454, (2005).

7. Z. Zhang, W. Li, W. Gong \& J. Zhong, An improved EEMD model for feature extraction and classification of gunshot in public places. 21st International Conference on Pattern Recognition (ICPR 2012) Tsukuba, Japan, 1517-1520, (2012). 